\title{
Signature Recognition based on Discrete Wavelet Transform
}

\author{
Sivana Salahadin Muhamed ${ }^{1}$, Muzhir Shaban Al-Ani' \\ ${ }^{1}$ University of Human Development, College of Science and Technology, Department of Computer Science, \\ Sulaymaniyah, Iraq, ${ }^{2}$ University of Human Development, College of Science and Technology, Department of Information \\ Technology, Sulaymaniyah, Iraq
}

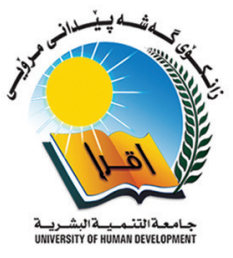

\section{A B S T R A C T}

Personal identification is an actively developing area of research. Human signature is a vital biometric attribute which can be used to authenticate human identity. There are many approaches to recognize signature with a lot of researches. The aim of this research is to introduce an efficient approach for signature recognition. This approach starts with the process the acquired signatures and stores these signatures in the database to be ready for verification. The collection of signature data based on collecting samples of 10 people and 10 signatures for each person through traditional ink stamp method. These signatures are digitized to be ready for processing. Many steps are applied to the acquired images to perform the pre-processing stage. The proposed approach based on discrete wavelet transforms to extract significant features from each signature image. Pre-processing is applied at the beginning of this approach to avoid any unwanted noise. This approach consists of many steps: Data acquisition, pre-processing, signature registration, and feature extraction. High recognition rate results $(100 \%)$ are obtained through applying this approach.

Index Terms: Biometrics System, Off-line Signature Recognition, Feature Extraction, Discrete Wavelet Transform

\section{INTRODUCTION}

Biometrics can be represented by physiological or behavioral characteristics for identification purposes. Physiological biometrics includes data derived directly from the measurement of any part of the human body, such as fingerprints, iris, retina, or facial shape [1]. Among the various behavioral characteristics that can be measured, the signature is recognized as one of the most reliable, unique, undeniable, and stable characteristics for human identification. Behavioral biometrics, in turn, analyses the data, which records the way a person behaves, such as the way of speaking or the dynamics of the signature [2].

\begin{tabular}{|l|l|}
\hline Access this article online \\
\hline DOI: 10.21928/uhdjst.v3n1y2019.pp19-29 & $\begin{array}{l}\text { E-ISSN: 2521-4217 } \\
\text { P-ISSN: 2521-4209 }\end{array}$ \\
\hline
\end{tabular}

Copyright (c) 2019 Muhamed and Al-Ani. This is an open access article distributed under the Creative Commons Attribution Non-Commercial No Derivatives License 4.0 (CC BY-NC-ND 4.0)
Signature recognition is one of the most important methods among the ways in which to authorize transactions and manifest the human identity compared with alternative strategies of electronic identification such as fingerprint scanning and retinal vascular pattern screening [3]. It's easier for folks to migrate from mistreatment the favored pen-and-paper signature to at least one wherever the written signature is captured and verified electronically, information about the way the human hand creates the signature such as hand speed and pressure measurements, acquired from special peripheral units, is needed [4].

Signature of someone is a vital biometric characteristic of a person's being and is employed for biometric authorization for many years [5]. While monetary establishments and different business organizations primarily specialize in the visual look of our signature, this leading to an accurate verification functions [6].

Signature recognition and verification system are widely applied for legal and common human identification using

Corresponding author's e-mail: Muzhir Shaban Al-Ani, University of Human Development, College of Science and Technology, Department of Information Technology, Sulaymaniyah, Iraq. E-mail: muzhir.al-ani@uhd.edu.iq

Received: 27-03-2019 Accepted: 16-04-2019 Published: 16-5-2019 
recognition and verification. The advantage of signature verification has a very high user acceptance in public because people are used the sign in their daily life [7].

Signature handwriting is a unique identity of every person, and cannot be lost, stolen or broken, also does not have user name and password in the case of forgetting password [8]. Wide range of applications is targeted within the field of electronic banking systems, electronic commerce, mobile applications, and security applications of significant installations $[9,10]$.

\subsection{Signatures Recognition Systems}

Handwritten signatures are simply divided into two categories:

\subsubsection{Online system}

The data are obtained using an electronic tablet and other electronic devices. They require a pen and a tablet connected to a computer to enter the dynamic information of the signature. The online approach will acquire additional data concerning the signature which has the dynamic properties of signature. Extract signature data concerned with many aspects: The writing speed, pressure points, strokes, and acceleration [11].

\subsubsection{Offline system}

Images of the signatures written on a paper are obtained using a scanner or camera, and then this signature takes as an input image to the system. The purpose of verifying the offline signature is to determine whether a signature is of a particular signer based on the scanned image of the signature and some images of the original signatures of the signer [12].

These wide applications give rise to a computerized signature verification system. However, in several systems, solely image of a signature is obtainable; therefore, the verification of offline signature appears additional aspects compared with the online signature verification system. The most significant issue is that a signature of an individual could vary in keeping with his/her mood, health, etc., in that even the real signer might not copy his/her signature as it is. In many cases, it seems somewhat difficult to distinguish between genuine signature and forgery signature [13].

\subsection{Levels of Forgeries}

Forgeries generally, based on making, forging, or fixing nearly any document, with the intent to different kinds of forgeries are classified into the subsequent categories [14]:

\subsubsection{Random forgery}

This type formed without any knowledge of the signer's name or signature shape. The name of the victim is used by the signer in his vogue to form a forgery signature called the easy forgery or random forgery. This forgery accounts for the bulk of the forgery cases though they are terribly straight forward to sight even by the eye [15].

\subsubsection{Unskilled forgery}

The person who made the signature with their vogue regarding that he has not any data of the orthography and knowledge. This will be through with observant the signature closely for the associate degree within enough time [16].

\subsubsection{Skilled forgery}

Undoubtedly, the foremost tough of all forgeries is formed by skilled cheaters or persons who have expertise in signature repetition. To detect this, one may either trace or imitate the signature by arduous manner. Various types of forgeries and related original signatures are shown in Fig. 1 [17].

\subsection{Signature Characteristics}

The signature should be treated as an image, because a person can use any symbol, letter, group of letters, etc., like signature. Therefore, it is possible not to separate the written letters, or maybe you cannot understand what is written. Therefore, the applied system for the analysis of signatures must use image processing ideas. Most likely, the signature of a signer may vary for each sign based on many factors. However, there must be a unique feature to identify the signature so that it can be used as biometrics. Below some of the essential features [18]:

- Invariant: It ought to be permanent over a protracted amount of time.

- Singular: It should be distinctive to each person.

- Inimitable: It should be inimitable by alternative suggests that.

- Reducible and comparable: It ought to be capable to be acceptable to a format that is simple to handle and digitally such as others.

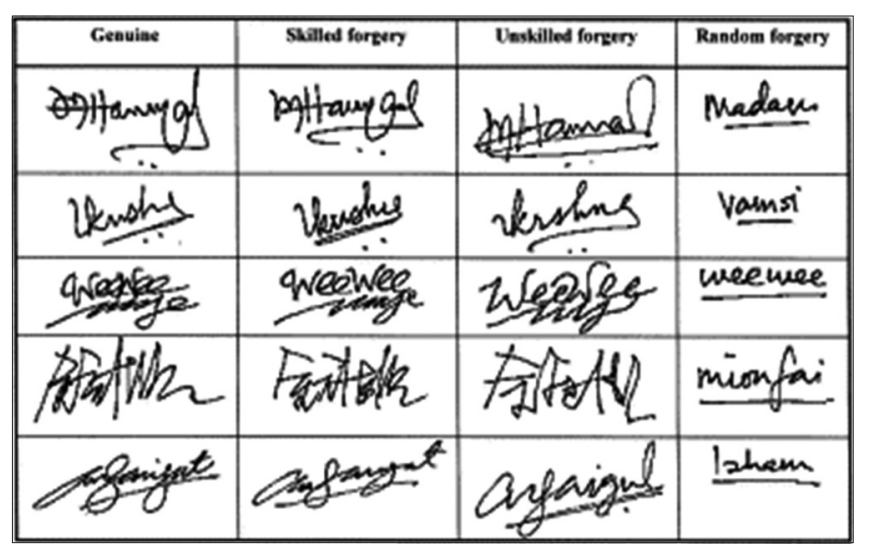

Fig. 1. Types of forgery. 
As signature conjointly belongs to at least one of the parameters that satisfy the characteristics; therefore, it is going to be used as a signal of person identification.

\subsection{Literature Review}

There are many methods that used for signature recognition; this section explains some of these methods:

Bharadi and Kekre (2010) showed a comprehensive review of handwriting recognition systems and special attention is paid to the analysis of static signature recognition systems (SRSs). They discussed a set of conventional features based on offline SRS, as well as cluster-based global characteristics. This system is multi-algorithmic; such systems combine the benefits of individual feature sets and improve recognition rates. The system reported a precision of $95.08 \%$, which is higher than the individual performance measures [19].

Tomar and Singh (2011) proposed a method to compare the performance of energy density methods; the directional characteristic method and the proposed directional method with the energy method, based on the time required for training, accuracy, acceptance, and rejection ratios. The obtained results showed that if the samples available for training are limited, then the method of directional characteristics is better than the density of the energy method but the merging of the directional characteristics with the energy method, leading to the best method. While the time required for training is a bit longer for this proposed method, accuracy and false acceptance rate (FAR) are satisfactory, and this additional time may be bearable. The obtained results indicated that False Rejection Ratio (FRR) varies randomly for all cases. In addition, this research gives the reason for future studies [20].

Radmehr et al. (2012) demonstrated that signature recognition poses a problem of recognition of difficult model. This research introduced a new SRS based on the transformation of Radon, shape dimension (fractal dimension), and support vector machine (SVM). Signature verification competition (SVC) database was used to evaluate the performance of this method. The results of the simulation indicate that the planned technique is promising in recognition of signatures. In addition, the performance of the SVM classification has been evaluated with different cores. This research presented an effective algorithm, and it may be valid in a large signature database where many signature styles are taken into account. Other attractive perspectives and challenges for future research are based on two aspects: How to extract more efficient features and how to combine an SVM-based classifier with other signature recognition methods [21].
Kanawade and Katariya (2013) explained that a person's signature is an important biometric attribute of a human being that can be used to authenticate human identity. However, human signatures can be treated as an image and recognized by artificial vision. They examined the problem of personal authentication through the recognition of signatures. Online and offline methods have been described in this work. The method of signature verification indicated that pre-processing and future extraction limits the advantage of being extremely acceptable to potential customers over the rest of the biometric solutions. This research indicated that verification and recognition of the offline identity are provided each time the signature is captured and delivered to the user in an image format. The signatures that are verified can support the parameters extracted from the signature using many image processing techniques. This research presented a case study of the signature verification method for offline signature verification [22].

Hiary et al. (2013) implemented an Arabic and Persian offline signature verification system. This technique is divided mainly into four stages: Pre-processing, recording of images, extraction of features and finally, verification of signatures. Pre-processing operations were performed to reduce processing time and increase accuracy. Then applied discrete wavelet transform (DWT) to extract the characteristics of the signature image and used logical operations with mathematical formulas to verify the signatures. The experimental results have shown reasonable improvements in many recent works. This approach reduces the number of levels of DWT levels and the amount of training required, with a low percentage of FAR of $1.56 \%$ and a false rejection rate of $10.9 \%$ and a low average error rate of $6.23 \%$. Future plans include improving this technique to increase the accuracy of verification and testing techniques with different forms and types of signatures [23].

Kanawade and Katariya (2013) explained a method to verify the handwritten signature in offline mode. This approach proposed a new technique of recognition and verification of signatures in offline mode based on global characteristics, mask, signature grid, and SVM. It is better to try counterfeiting using the online signature verification method. The concept of offline signature verification can be extended to other biometric identification systems, such as handwriting. Combined signature features with other biometric aspects such as speech and facial recognition; it provides better results than any individual system [24].

Bhattacharya et al. (2013) implemented a signature authentication machine to provide a security system of 
simple, safe, and fast biometric behavior. Based on some mathematical coordinates, this methodology is faster than the different forms. Color technique is applied to make it safer. The interface of this application is extremely simple, which makes it easy to use and easy user-friendly. The implemented system has some limitations: It can only identify static changes in a signature and cannot identify any dynamic change in a signature. This signature authentication system works only for offline mode, and the results were tested only offline. The proposed system for recognizing and verifying offline signatures is implemented through pixel matching technique (PMT). PMT is used to verify the signature of the user with the signature stored in the database. The performance of the proposed method was compared with the existing back propagation artificial neural network and SVM [25].

Doroz et al. (2014) proposed an efficient method of verification of handwritten signatures. This method creates complex functionalities for each signature that describe this signature. These characteristics are based on the analysis of the dependencies between the dynamic characteristics recorded by the tablets. These complex features are used to create vectors that describe the signature. The elements of these vectors are calculated using the measures indicated in this research. The similarity between the signatures was evaluated by determining the similarity of the vectors in the comparative signatures. The obtained results showed a high effectiveness of the verification using the proposed method where the precision obtained was $96.67 \%$ [26].

Malik and Arora (2015) talked about two types of signature verification system are present offline (static) and online (dynamic). The biometrics has an important advantage over traditional authentication techniques due to the fact that biometric characteristics of the individual are not easily acceptable for convert, are unique of every person, and cannot be lost, stolen, or broken. The signature is acquired as an image; this image shows a personal style of human handwriting. A signature verification system sometimes focuses on the detection of one or more type of forged signatures. The purpose of this work is to design and develop an offline handwritten signature verification system that will differentiate between the original signature and closer forge signature depending on some special features [27].

Al-Ani and Al-Saidi (2011) studied how to improve handwritten Arabic signature recognition. They this work proposed SRS used for many applications and have played an important role in these applications. This work presented a simple and efficient signature recognition approach based on the discrete Fourier transform used to extract features. Many people are registered through a built-in signature database. The results obtained indicated that a good and effective recognition rate was recognized, as the results showed, it provides value almost greater than zero [28].

Hafemann et al. (2015) proposed a method for offline signature verification. Although the categorization of authentic signatures and qualified counterfeits remains a difficult task, the error rates have decreased significantly in recent years, mainly due to the progress of deep learning applied to the task used for the verification of Persian firms of convolutional neural network (CNN). Analyzing the recent contributions to the field, they can notice that they concentrate in the following categories: Obtaining better features, improving classification with a limited number of samples, augmenting the datasets, and building model ensembles. As a suggestion for future work, this research can be continued to explore higher feature representations and introducing methods to improve classification with a limited number of samples [29].

Hedjaz et al. (2018) proposed an offline signature recognition method using binary features. It is clear; the offline signatures recognition is mainly important for the authentication of administrative and official documents that required greater precision. The characteristics are extracted using two descriptors: Binary statistical image and local binary models. To evaluate the reliability of the method, experiments were conducted using MCYT-75 and GPDS-100 databases. Using a nearest $\mathrm{k}$-neighbor classifier, the recognition performance reaches a high $97.3 \%$ and $96.1 \%$ for the MCYT-75 and GPDS-100 databases, respectively. During the verification of the signature, the accuracy of the classification measured with the same error index equal error rate (EER) reached, respectively, $4.2 \%$ and $4.8 \%$ in the GPDS-100 and the GPDS- 160 . In addition, the EER for the MCYT-75 database reached 7.78\% [30].

\subsection{Statement of the Problem}

Many researches are published regarding signature recognition issue but many of these researched suffered from many aspects. This paper presents an offline SRS. DWT is suggested to extract common features and different change of the original image and signal of signature. We expected an efficient result in signature recognition that supports the verification step.

\section{METHODOLOGY}

This section can be divided into two parts: Data collection and implemented algorithm. 


\subsection{Data Collection}

The offline signature images are acquired from traditional papers. This requires a high specific signature image that can be achieved through high-resolution scanner. In general, the first step of an offline signature verification system is to extract these signatures from traditional papers using a scanner. In this research, signatures are collected using either black or blue ink on a white A4 paper. Each A4 paper is divided into 10 parts to cover 10 signatures per page. A group of 100 persons are used to collect 10 signatures from each person, which resulting 1000 signature samples as shown in Fig. 2.

\subsection{Implemented Approach}

The offline signature approach for verification and recognition is divided into many steps as shown in Fig. 3:

1. Data acquisition: This step including converting signature images into digital image using a scanner device.

2. Pre-processing: This step including removing unwanted noise from signature images where that negatively influences the accuracy of verification and recognition.

3. Signature registration: In which the signature is scaled into an appropriate form to realize the higher and correct result.

4. Feature extraction: In which extract common features based on DWT.

5. Signature verification: In which compare the input with the original image and the result to show the accuracy of the system.

\section{RESULTS AND DISCUSSION}

To explain the obtained results regarding the implemented approach, we will pass the steps of this approach gradually.

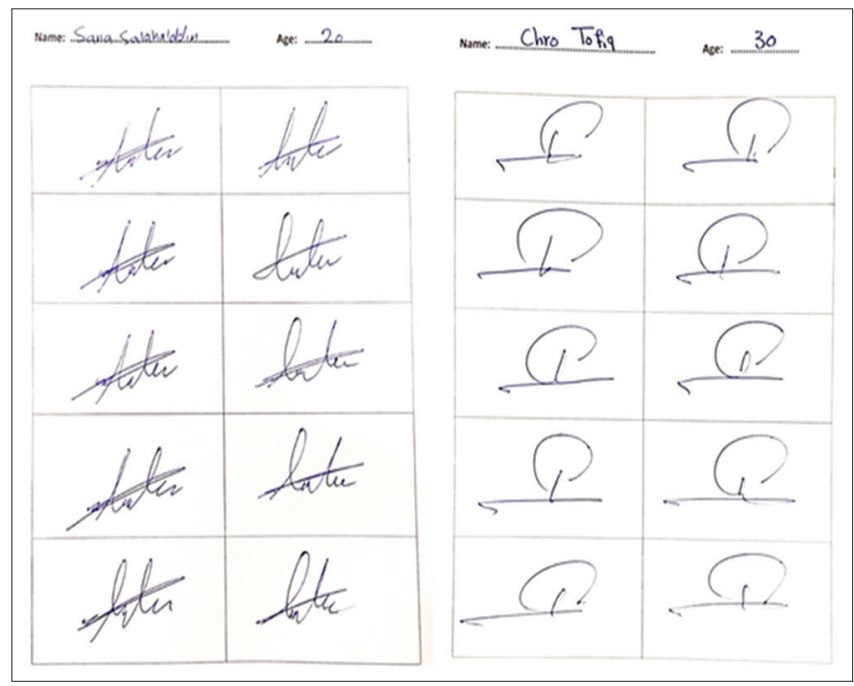

Fig. 2. Sample of signatures.

\subsection{Pre-processing}

Pre-processing step plays an important role in signature recognition to overcome any raised problem. The purpose of this step is to make standard signatures that are ready for the feature extraction process. In this research, the pre-processing step is achieved through the following operations.

\subsubsection{Noise removal}

Minimizing of noise is an important operation that affects the overall signature recognition process because the signature image may contain unwanted information. In this research, the implemented noise removal operation is performing through filtering that not affects the details of signature image information. The median filter is an adequate filter that resolves the problems of noise reduction. Both signature images before and after applying media filter are shown in Figs. 4 and 5, respectively.

\subsubsection{Image filtering and binarization}

The true color image red green blue (RGB) is converted into grayscale intensity image through eliminating the hue and saturation information while retaining the luminance element. A grayscale image could be an information matrix whose values represent intensities within some range wherever every part of the matrix corresponds to at least one image pixel.

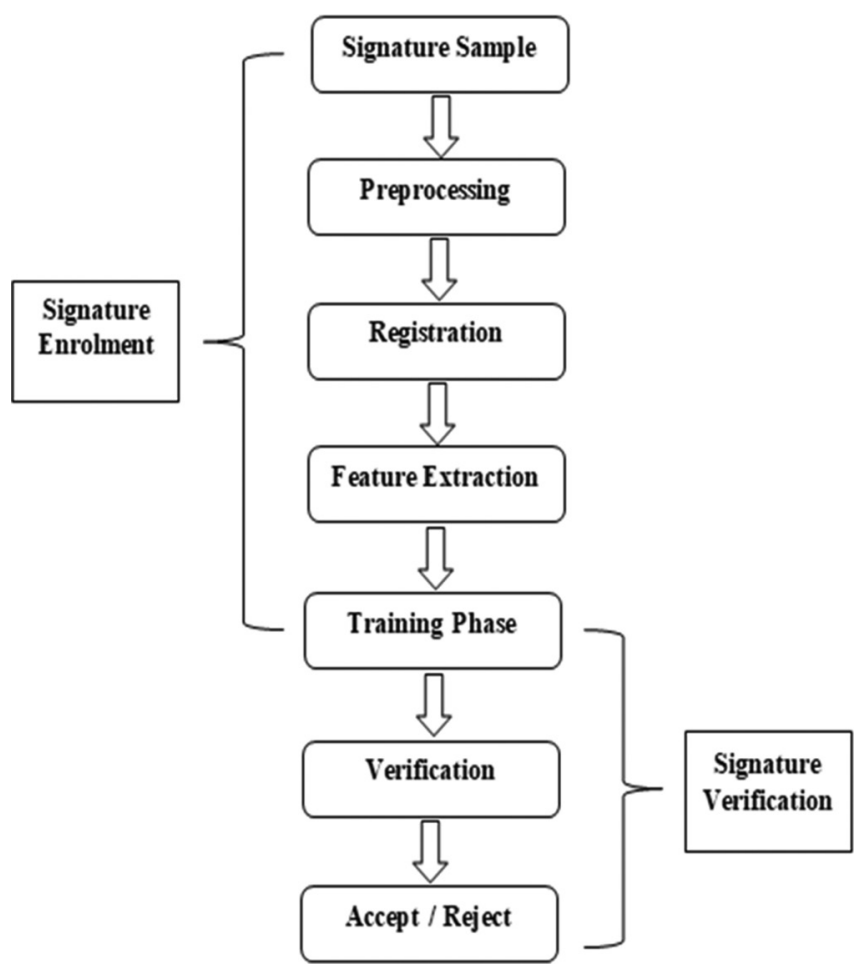

Fig. 3. Signature verification and recognition system. 
The process of signature image filtering and binarization is shown in Figs. 6 and 7, respectively.

\subsubsection{Width normalization}

Signature dimensions could have intrapersonal and interpersonal variations. That the image width is adjusted

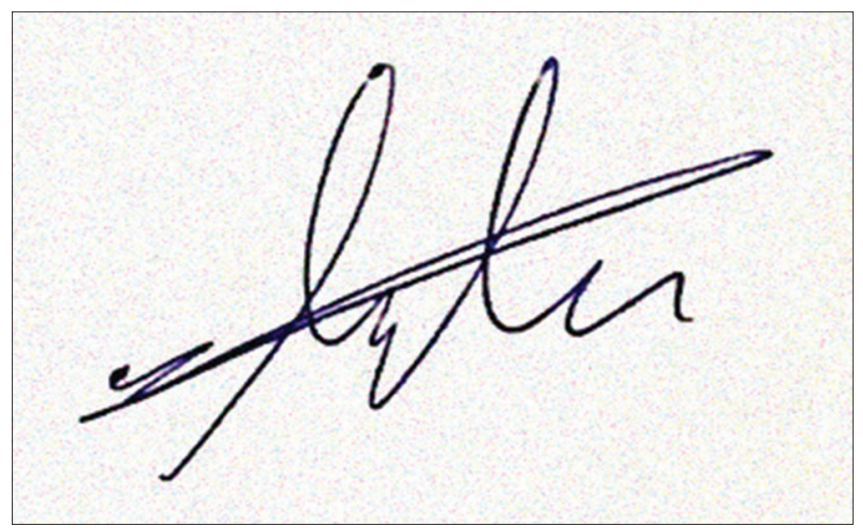

Fig. 4. Signature image before noise removal.

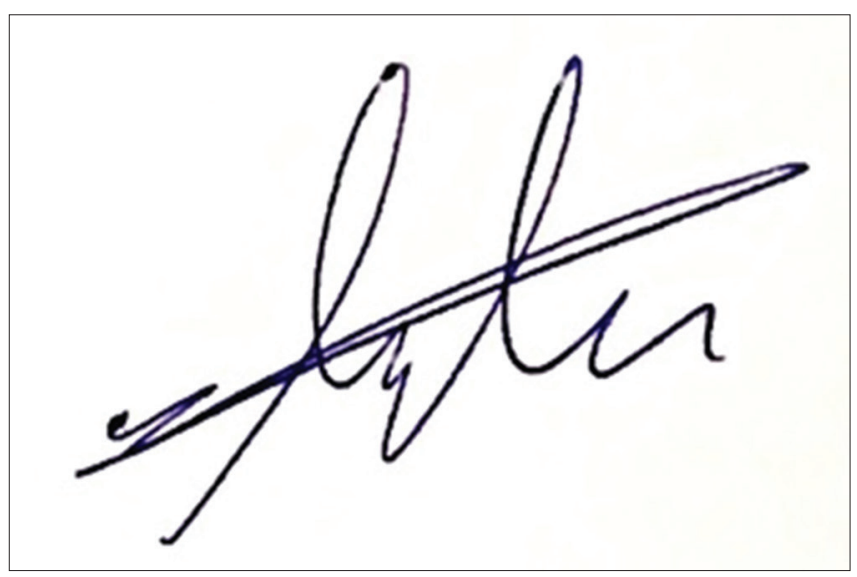

Fig. 5. Signature image after noise removal.

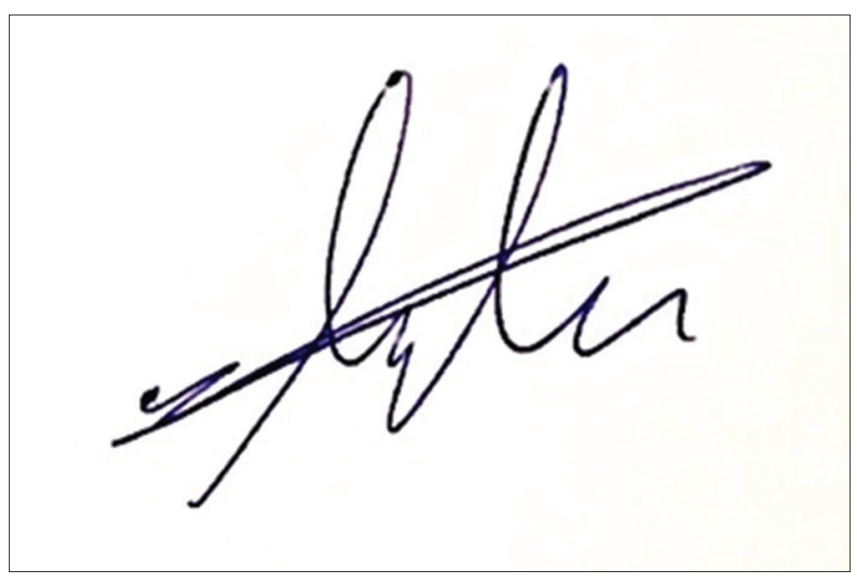

Fig. 6. Signature image before binarization. to a default value and also the heights can be modified considering constant height-to-width ratio, as a result, the width dimension is adjusted to 100. Signature image before and after normalization is shown in Figs. 8 and 9, respectively.

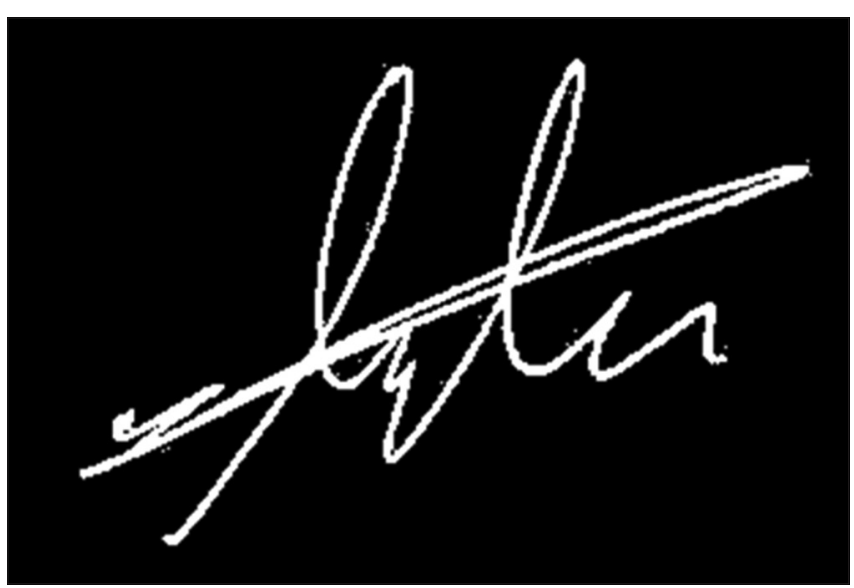

Fig. 7. Signature image after binarization.

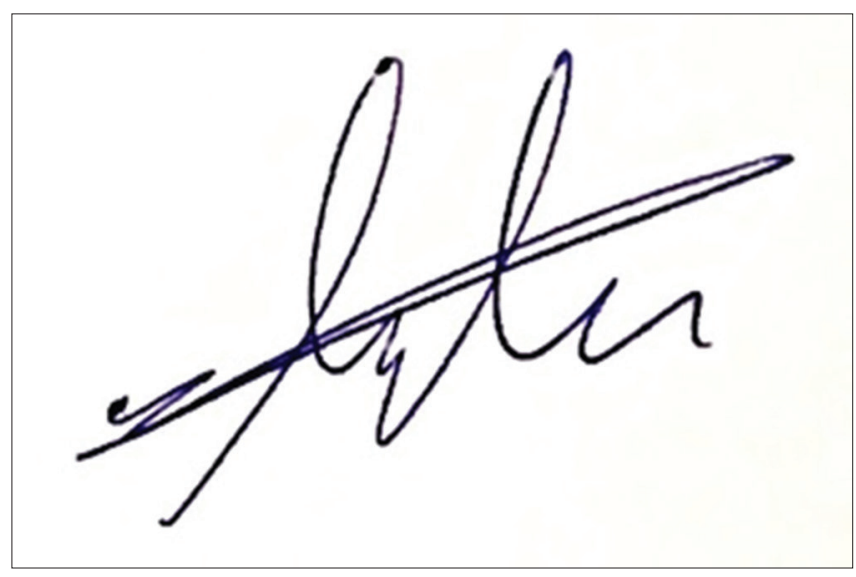

Fig. 8. Signature image before normalization.

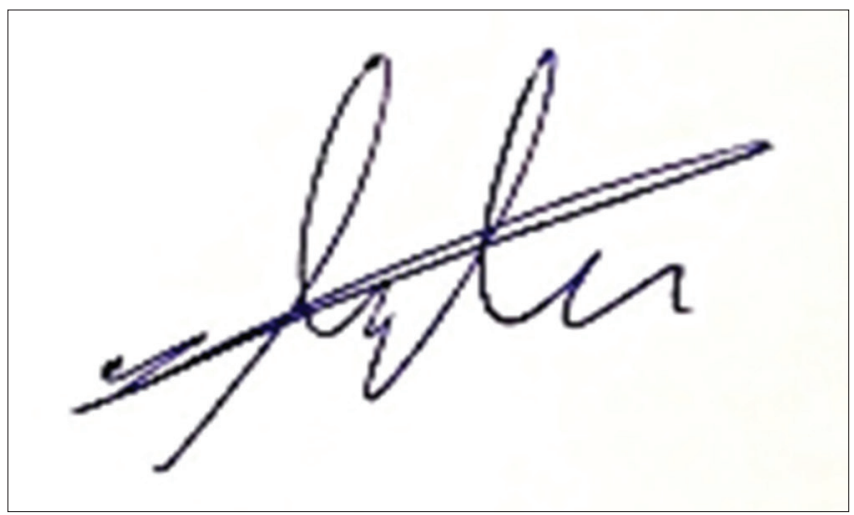

Fig. 9. Signature image after normalization.

UHD Journal of Science and Technology | Jan 2019 | Vol 3 | Issue 1 


\subsubsection{Thinning}

The goal of the thinning operation is to eliminate the thickness variations of a pen by creating the line of one pixel of thickness that leading to accurate recognition rate. Furthermore, signatures are thinned so as to reduce the computations needed by the graph matching algorithm. Figs. 10 and 11 represent signature images before and after thinning.

\subsection{Registration}

The registration step contains two main operations; scaling and shifting. In scaling, the signature is re-scaled into the suitable type to realize the higher and accurate result. After that, shifting operation is applied to determine the center of the signature. These operations are mentioned below [5].

\subsubsection{Scaling}

This step resizing all the signature images for all persons. Alternative techniques performed this resizing supported the maximum height and width for all samples in the database.

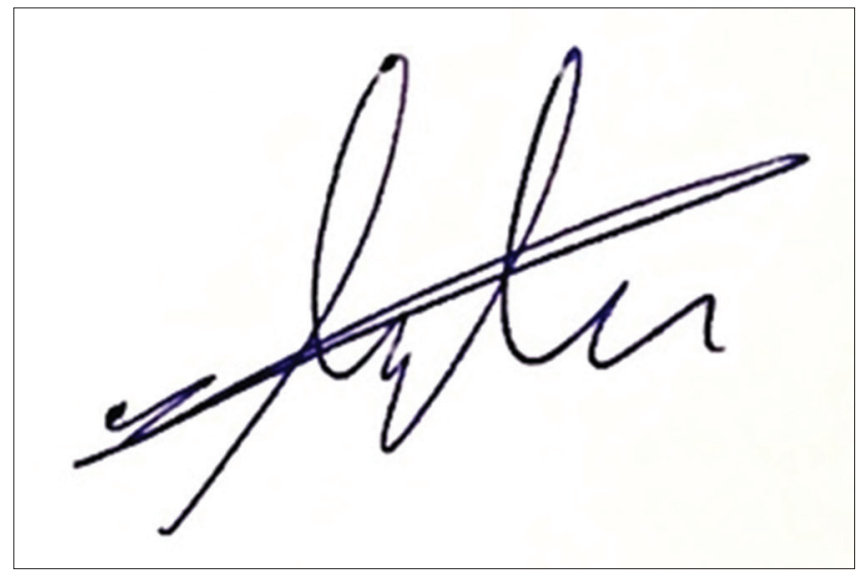

Fig. 10. Signature image before thinning.

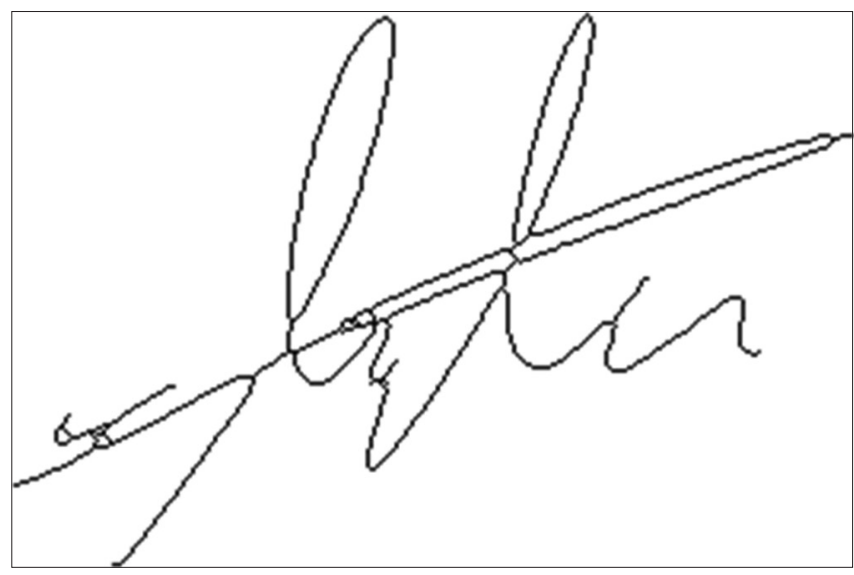

Fig. 11. Signature image after thinning.
This leading to unnecessary, superfluous stretching of signatures and may cause errors in verification. To avoid this problem, the implemented technique uses the maximum height and width for signatures of the same person rather than unifying over all dataset where Figs. 12 and 13 show the signature images before and after scaling.

\subsubsection{Shifting}

In this step, the scaled signature image is aligned to the center of gravity of each person's signatures. Figs. 14 and 15 show the signature images before and after applying to the align process.

\subsection{Feature Extraction}

Feature extraction is an important step to enhance the signature image characteristics that are necessary to apply the comparison operation. DWT is to extract the perfect features before the verification step. The advantage of using vectors is the fact that they always have the same length, which does not depend on the size of the signature.

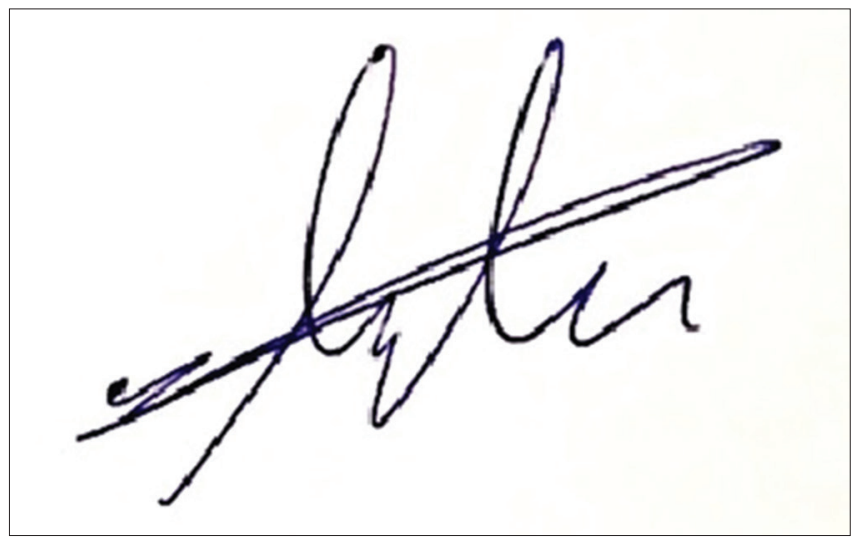

Fig. 12. Signature image before scaling.

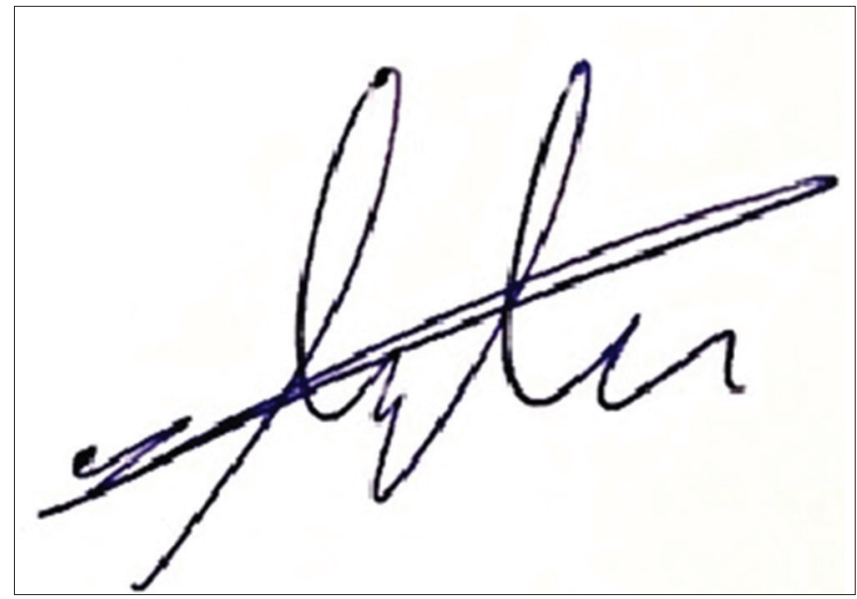

Fig. 13. Signature image after scaling. 


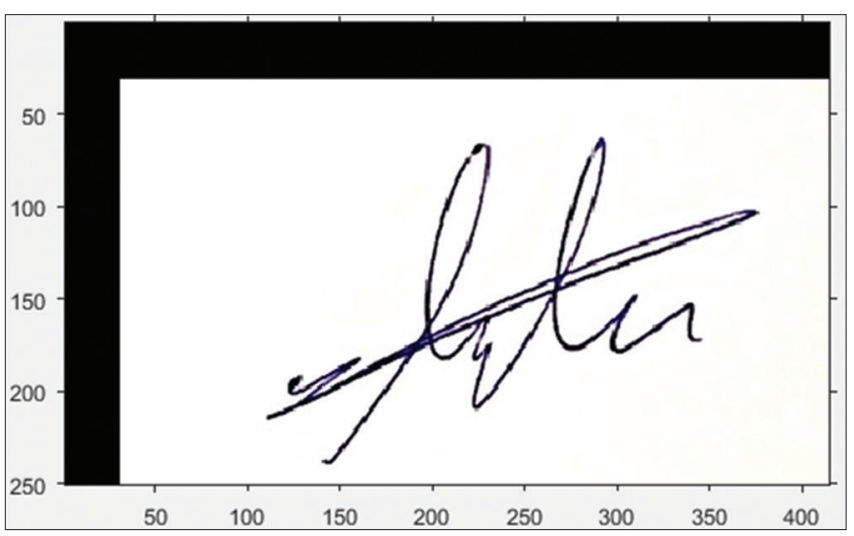

Fig. 14. Signature image before alignment.

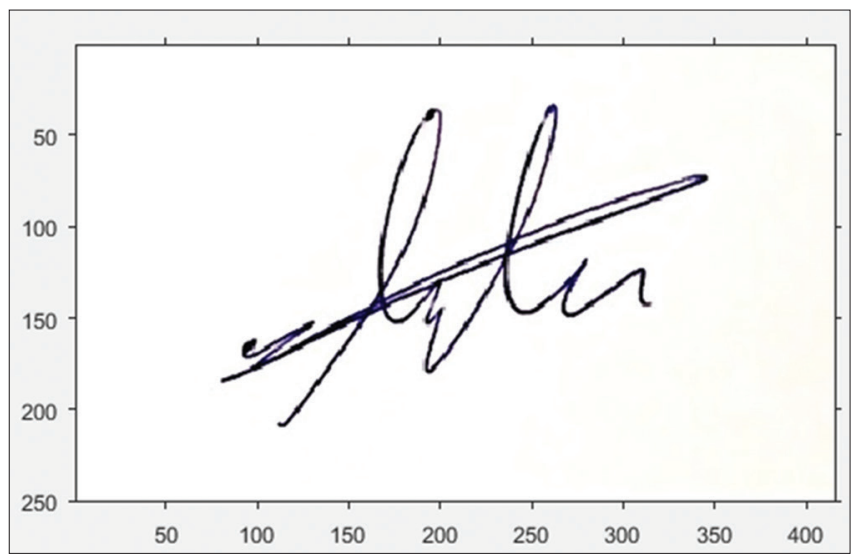

Fig. 15. Signature image after alignment.

DWT produces multi-scale image decomposition using filtering and sub-sampling that leading to the shape of the decomposition image where terribly effectively revealing knowledge redundancy in many scales.

Since signature images are two-dimensional (2D) signals, so it is better to increase the theme to $2 \mathrm{D}$ space by applying the transformed row and column-wise, respectively. According to 2D DWT, four sub bands are generated for each level of DWT. One low-pass sub-band containing the coarse approximation of the source image referred to as low-low (LL) sub-band, and three high-pass sub-bands that exploit image details across totally different directions, high-low for horizontally, low-high for vertical, and high-high ( $\mathrm{HH})$ for diagonal details, as shown in Fig. 16.

Applying DWT, each sample of the signature image is decomposed into four images, the primary image represents the LL band values, whereas the other three images represent the $\mathrm{HH}$ in vertical, diagonal, and horizontal directions,

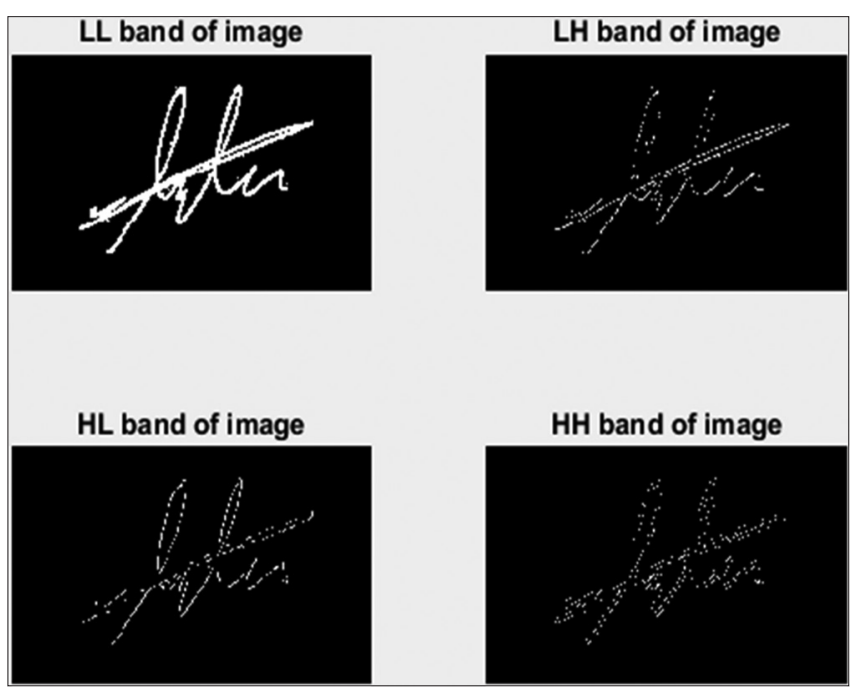

Fig. 16. Discrete wavelet transform bands: Low-low, low-high, highlow, and high-high.

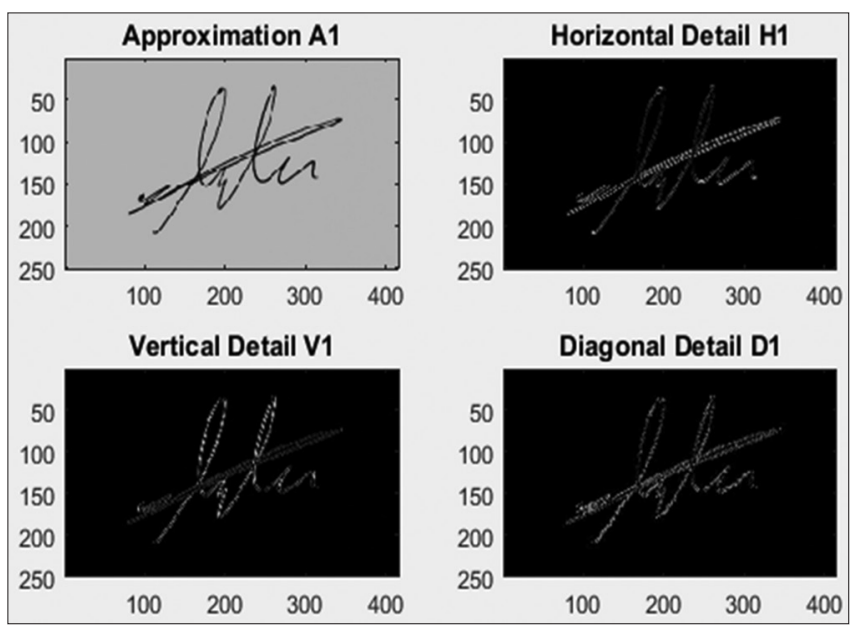

Fig. 17. Discrete wavelet transform, (A1) approximation results, (V1) vertical, (H1) horizontal, and (D1) diagonal high pass results.

respectively, as shown in Fig. 17. DW' is especially used to extract the features from the signature image. The proposed technique uses high pass images to extract the mandatory data for signature verification.

The DW'T of a signal is calculated by passing it through a series of filters. First the samples are passed through a low-pass filter with impulse response resulting in a convolution of the two:

$$
y[n]=(x * g)[n]=\sum_{k=-\infty}^{\infty} x[k] g[n-k]
$$

The signal is additionally rotten at the same time using a highpass filter. The outputs are given the brief coefficients (from 

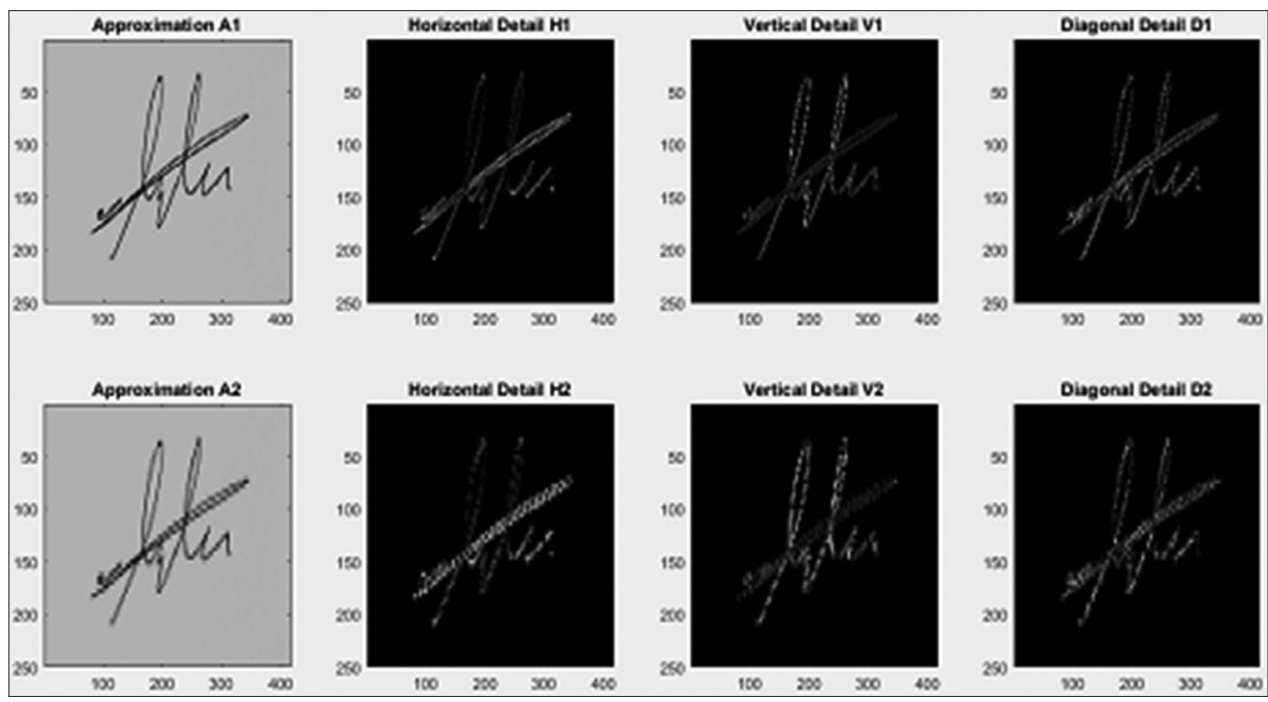

Fig. 18. Different between two levels of discrete wavelet transform.

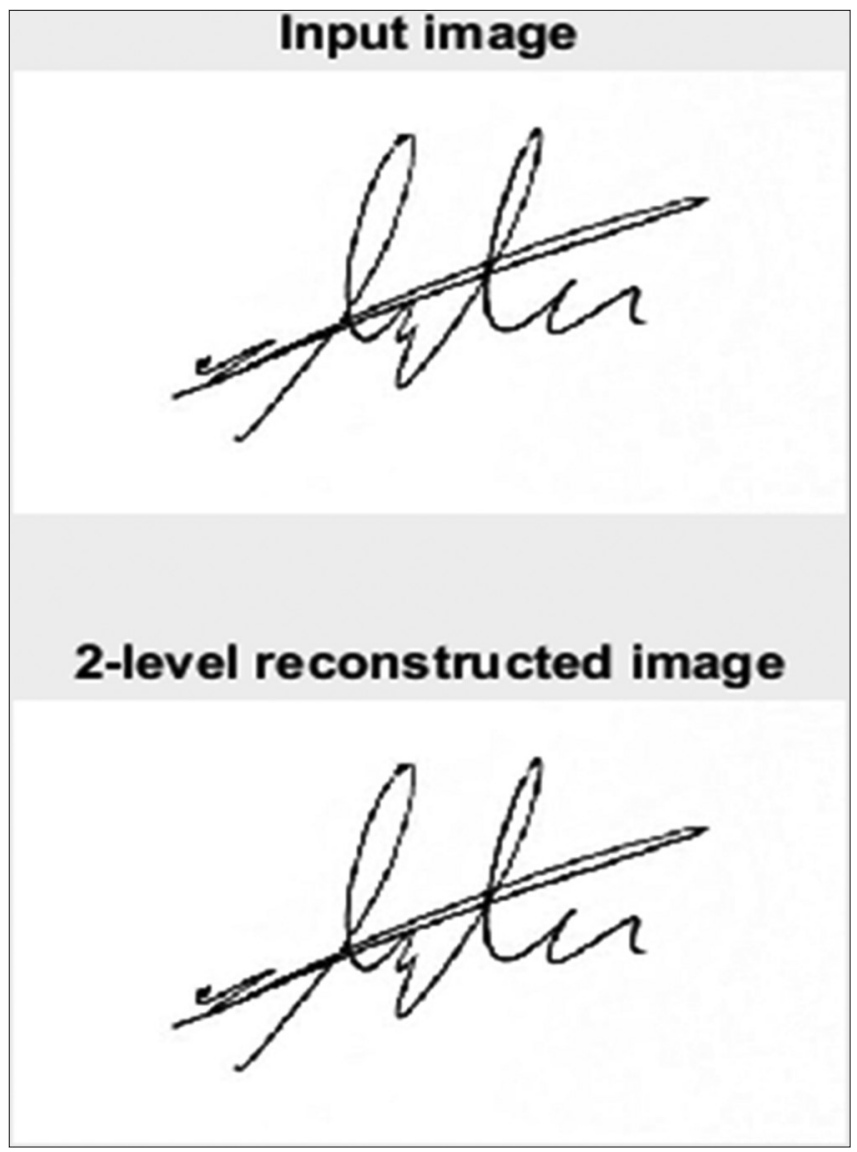

Fig. 19. Original image and 2-level reconstruct the image.

the high-pass filter) and approximation coefficients (from the low-pass). It is necessary that the two filters are associated with one another and that they are called a construction mirror filter.

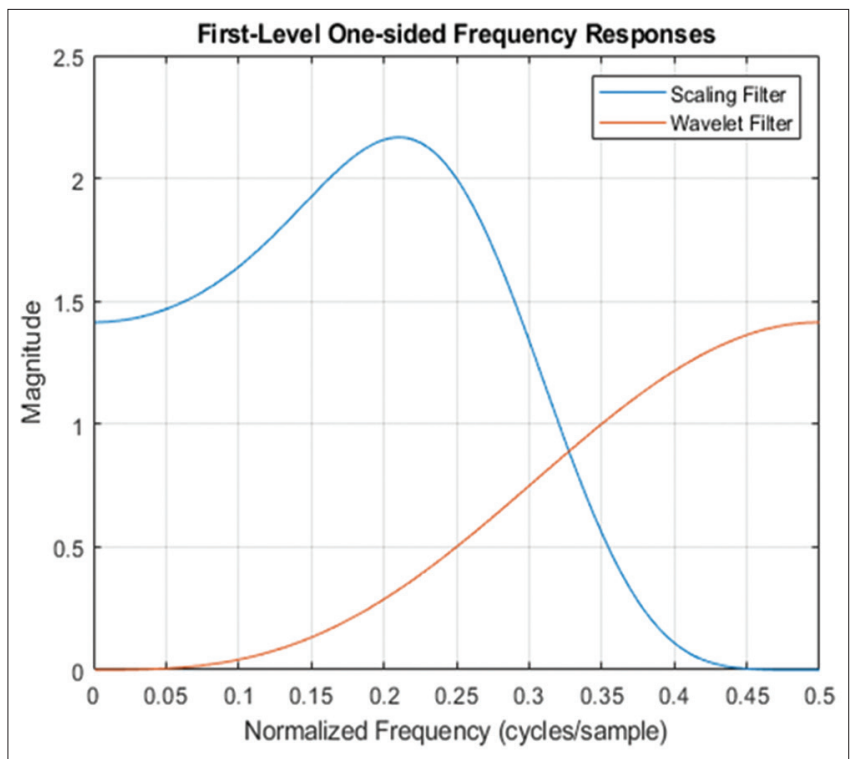

Fig. 20. First-level one-side signal filtering and scaling.

Each sample is changed during the decomposition first level and decomposition second level, the primary image represents the low pass values, whereas the opposite three images represent the first level high pass in vertical, diagonal, and horizontal directions, and the second level of primary image represents the low pass values whereas the opposite three images represent the second level high pass in vertical, diagonal, and horizontal directions. Fig. 18 illustrates the differences between the two levels of the same sample.

Fig. 19 represents two levels reconstructed signature image compared with the original image. 


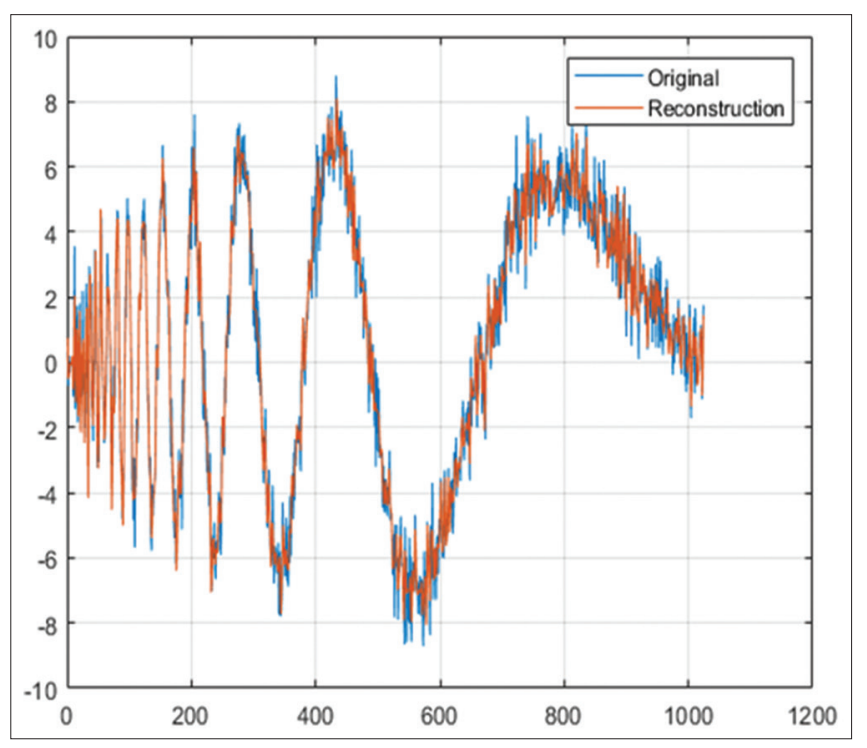

Fig. 21. Original signal compared with reconstruct signal.

Fig. 20 shows the obtained first-level one side DWT of a noisy Doppler signal using the wavelet (high pass) and compared that with the scaling (low pass) filter.

Fig. 21 shows the reconstructed of the smoothed version of the signal using the approximation coefficients compared with the original signal, in which a high similarity is obtained.

\section{CONCLUSIONS}

Signature recognition is one of the most widely accepted personal attributes for human biometric identification. In this paper, we proposed and discussed offline signature recognition based on DW'T. The proposed approach is mainly divided into three steps: Pre-processing, image registration, and feature extraction. Pre-processing operations were performed to extend the accuracy and reduce processing time. This approach is performed using many levels of DW'T to extract effective features from the signature image. An efficient recognition rate reached to $100 \%$ is obtained through implementing the proposed approach.

\section{REFERENCES}

[1] IGI Global. Biometrics: Identification and Security. In: "Multidisciplinary Perspectives in Cryptology and Information Security". IGI Global, Pennsylvania, USA, 2014.

[2] M. S. Al-Ani and Z. A. Kakarash. "Future aspects of intelligent car parking based on internet of things". UHD Journal of Science and Technology, vol. 2, no. 1, pp. 19-26, 2018.
[3] M. S. Al-Ani. "Electrocardiogram waveform classification based on P-QRS-T wave recognition". UHD Journal of Science and Technology, vol. 2, no. 2, pp. 1-6, 2018.

[4] S. Impedovo and G. Pirlo. "Verification of Handwritten Signatures : An Overview". 14 $14^{\text {th }}$ International Conference on Image Analysis and Processing. ICIAP, Modena, Italy, 2007.

[5] A. Karouni, B. Daya and S. Bahlak. "Offline signature recognition using neural networks approach". Procedia Computer Science, vol. 3, pp. 155-161, 2010.

[6] M. Arathi and A. Govardhan. "An efficient offline signature verification system". International Journal of Machine Learning and Computing, vol. 4, no. 6, pp. 533-536, 2014.

[7] M. S. Al-Ani. "Study the characteristics of finite impulse response filter based on modified kaiser window". UHD Journal of Science and Technology, vol. 1, no. 2, pp. 1-6, 2017.

[8] V. Malik and A. Arora. "Signature recognition using matlab". International Journal for Research in Applied Science and EngineeringTechnology (IJRASET), vol. 3, no. 6, pp. 682-687, 2015.

[9] B. M. Thuraisingham, L. Khan, M. Masud and K. W. Hamlen. "Data Mining for Security Applications". Conference: 2008 IEEE/IPIP International Conference on Embedded and Ubiquitous Computing (EUC 2008), Shanghai, China, December 17-20, 2008.

[10] S. Soviany, S. Puscoci, V. Sandulescu and C. Soviany. "A biometric security model for mobile applications". International Journal of Communications, vol. 3, pp. 85-92, 2018.

[11] M. Al-Ani. "Biometric Security". In: Handbook of Research on Threat Detection and Countermeasures in Network Security. IGI Global, Pennsylvania, USA, 2015.

[12] A. M. Omar, N. M. Ghanem, M. A. Ismail and S. M. Ghanem. "Arabic-Latin Offline Signature Recognition Based on Shape Context Descriptor". International Conference on Computer Vision Systems, ICVS: Computer Vision Systems, Berlin, pp 24-33, 2015.

[13] R. Jana, R. Saha and D. Datta. "Offline signature verification using euclidian distance". International Journal of Computer Science and Information Technologies, vol. 5, no. 1, pp. 707-710, 2014.

[14] H. M. El-bakry and N. Mastorakis. "Personal Identification Through Biometric Technology". AIC'09 Proceedings of the $9^{\text {th }}$ WSEAS International Conference on Applied Informatics and Communications, Moscow, Russia, pp. 325-340, 2009.

[15] S. Pal, M. Blumenstein and U. Pal. "Automatic off-Line Signature Verification Systems: A Review". IJCA Proceedings on International Conference and Workshop on Emerging Trends in Technology. ICWET, Mumbai, vol. 14, pp. 20-27, 2011.

[16] S. Mushtaq and A. H. Mir. "N Signature Verification: A Study". $4^{\text {th }}$ International Conference on Computer and Communication Technology. ICCCT, 2013.

[17] S. Bhatia, P. Bhatia, D. Nagpal, S. Nayak. "Online signature forgery prevention". International Journal of Computer Applications, vol. 75, no. 13, pp. 21-29, 2013.

[18] M. A. Maurello, J. A. Clarke and R. S. Ackley. Signature characteristics in contact calls of the white-nosed coati". Journal of Mammalogy, vol. 81, no. 2, pp. 415-421, 2008.

[19] V. A. Bharadi and H. B. Kekre. "Off-line signature recognition systems". International Journal of Computer Applications, vol. 1, no. 27, pp. 48-56, 2010.

[20] M. Tomar and P. Singh. "An Intelligent Network for Offline Signature Verification Using Chain Code". International Conference on Computer Science and Information Technology CCSIT: Advanced Computing. CCSIT, Bangalore, pp. 10-22, 2011. 
[21] M. Radmehr, S. M. Anisheh, M. Nikpour and A. Yaseri. "Designing an offline method for signature recognition". World Applied Sciences Journal, vol. 13, no. 3, pp. 438-443, 2011.

[22] M. V. Kanawade and S. S. Katariya. "Signature verification and recognition case study". International Journal of Electronics, Communication and Instrumentation Engineering Research and Development, vol. 3, no. 1, pp. 77-86, 2013.

[23] H. Hiary, R. Alomari, T. Kobbaey, R. Z. Al-Khatib, M. A. Al-Zu'Bi and $\mathrm{H}$. Hasan. "Off-line signature verification system based on DWT and common features extraction". Journal of Theoretical and Applied Information Technology, vol. 51, no. 2, pp. 165-174, 2013.

[24] M. V. Kanawade and S. S. Katariya. "Review of offline signature verification and recognition system". International Journal of Emerging Technology and Advanced Engineering, vol. 3, no. 7, pp. 659-662, 2013.

[25] I. Bhattacharya, P. Ghosh and S. Biswas. "Offline signature verification using pixel matching technique". Procedia Technology, vol. 10, pp. 970-977, 2013.

[26] R. Doroz, M. Palys, T. Orczyk and H. Safaverdi. "Method of signature recognition with the use of the complex features". Journal of Medical Informatics and Technologies, vol. 23, pp. 1-8, 2014.

[27] V. Malik, A. Arora. "Signature Recognition Using Matlab". International Journal for Research in Applied Science and Engineering Technology (IJRASET), vol. 3, no. 4, 2015.

[28] M. S. Al-Ani and M. M. Al-Saidi. "An improved proposed approach for handwritten arabic signature recognition". Advances in Computer Science and Engineering, India, vol. 7, no, 1, pp. 25-35, 2011.

[29] L. G. Hafemann, R. Sabourin and L. S. Oliveira. "Offline Handwritten Signature Verification Literature Review". Computer Vision and Pattern Recognition, (cs.CV); Machine Learning (stat. ML), IPTA, 2015.

[30] H. Hedjaz, R. Djemili and B. Hocine. "Signature recognition using binary features and KNN". International Journal of Biometrics, vol. 10, no. 1, pp. 1-5, 2018. 